\section{Visual discrimination between rectangular and nonrectangular parallelopipeds*}

\author{
DAVID N. PERKINS $†$ \\ Project Zero, Harvard University, Cambridge, Massachusetts 02138
}

Twenty-seven university siudents judged whether each of 128 drawings of parallelopipeds appeared to represent three-dimensional rectangular boxes. Half the pictures could not geometrically have been projections of rectangular boxes. The null hypothesis that Ss' judgments were unrelated to geometry was rejected at the .001 level of significance, and the correlation between Ss' judgments and perfect discrimination averaged .86 over three variations of the experiment. The results support a general hypothesis about the perception of simple space forms according to which viewers impose geometric constraints, such as rectangularity and symmetry, but only when the constraints are projectively possible.

The human viewer readily interprets line drawings of simple solid forms as representing three-dimensional shapes, in spite of the absence of numerous traditional depth cues such as stereo disparity, occlusion, texture gradients, and parallax. From a geometric standpoint, the lines on the page could be the projection of any one of an infinity of spatial configurations. One's visual impression, however, is generally of a fairly stable and specific shape, not of a shifting exploration along a continuum of possibilities. Whether this impression is well founded, and on what principles the eye selects a particular shape from the continuum are the key questions.

Perkins (1971) offered a general hypothesis about the perception of simple spatial forms, proposing that the visual system resolves projective ambiguity by imposing various geometric regularities such as symmetry, rectangularity, and the parallelness of planes in space. The visual system combines these constraints with the requirement that its spatial interpretation indeed project to the stimulus image, and proceeds to infer a specific space shape and orientation.

This proposal relates to the research on the covariation of perceived shape and slant initiated by Beck and Gibson (1955). Developing a suggestion of Koffka's, they pointed out that only certain geometrically determined combinations of shape and slant of a surface fragment could yield a given stimulus image. Their experiments

\footnotetext{
*This research was conducted at Project Zero, Harvard Graduate School of Education, operating under National Science Foundation Grant No. GB-31064 and Office of Education Grant No. OEG-0-9-31 0283-3721 (010).

† A ppreciation is extended to Helen Bray, Nanette de Maine, Ennio Mingolla, Louisa Rogoff, and Roy Rudenstine, for their assistance throughout, and to Howard Gardner, for his insightful comments on the experimental design and on the manuscript.
} constraints that eliminate the projective ambiguity remaining in the shape/slant covariation as there is with that covariation itself. Third, a new means of assessing the visual system's respect for projective relations is introduced: viewers' judgments as to whether particular additional constraints are projectively possible at all for a given stimulus. This will become clearer in the examples of the following paragraphs.

This paper reports an initial test of the hypothesis, turning on two points: first, that not all box-like drawings can geometrically be projections of rectangular boxes, and second, that viewers tend to "impose on" or "read into" pictures a rectangular organization, but only when this is geometrically appropriate (Perkins, 1968). Thus, Fig. 1, which could be a projection of a rectangular object (the criterion is described below), is seen as rectangular. But Fig. 2, which could not, is interpreted in three alternative ways, with Angles $A$ and $B$ right but $C$ obtuse, with $B$ and $C$ right but $A$ acute, or with $A$ and $C$ right but $B$ obtuse.

To examine this phenomenon carefully, an experiment was designed in which Ss judged whether a series of pictured boxes appeared rectangular. The experiment examined the general hy pothesis described above in terms of two particular questions: (1) Did Ss indeed only judge a picture to appear rectangular when it could projectively be so, and (2) when a picture could be rectangular, did Ss indeed judge it to be so? The two are logically independent, for if a picture projectively could not be rectangular, the visual system might ignore this

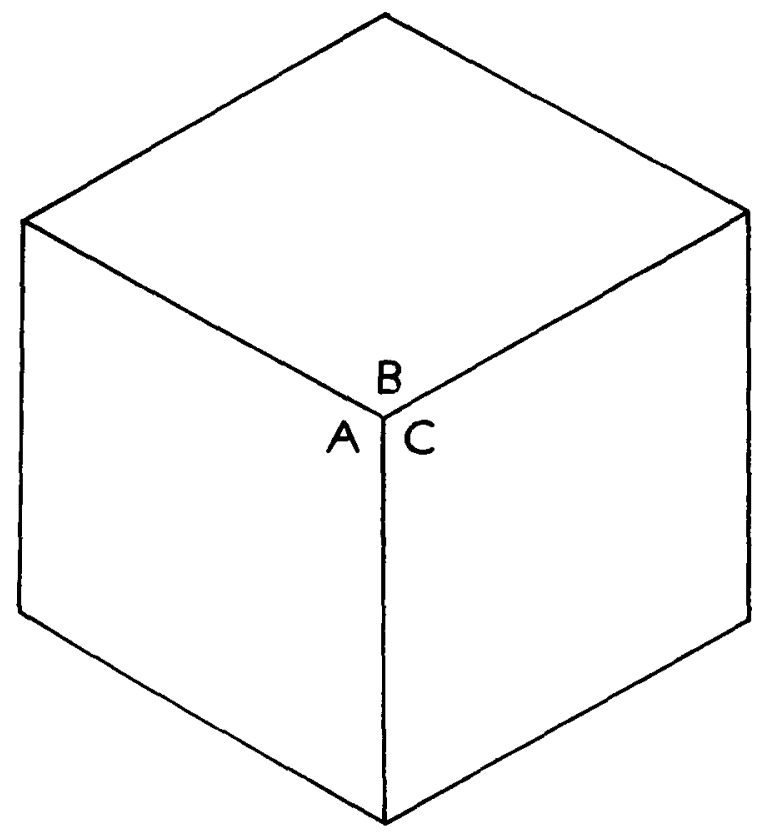

Fig. 1. A box that appears rectangular. 


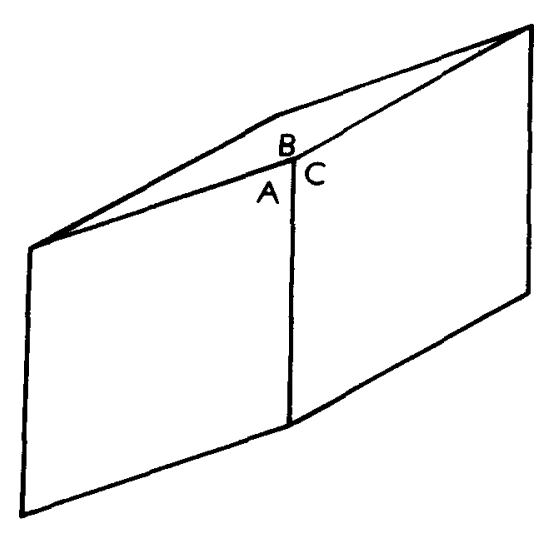

Fig. 2. A box that does not appear rectangular.

constraint; and if a picture could be rectangular, this is not to say that it must be so interpreted.

Whether a picture can geometrically be a projection of a rectangular solid is an all-or-none matter. Inevitably, there are some rectangular pictures arbitrarily close in their measurements to some nonrectangular pictures, although there are also pictures far from any picture of the opposite category. Accordingly, one could hardly expect perfect discrimination between the two kinds. The hypothesis could at best stand as an idealized description of behavior.

The stimuli were selected to range over pictures close to and far from the opposite category so that the experiment would determine the profile of viewers' discriminative capacities. Especially sharp differentiation, that is, mostly rectangular judgments of rectangular pictures and nonrectangular judgments of nonrectangular pictures, would provide evidence for both the behavior patterns, 1 and 2 , as idealized descriptions. But, if viewers did not respect projective possibility at all, there should be as many rectangular judgments of nonrectangular pictures as of rectangular pictures. And if viewers did not persistently exercise rectangularity as an organizing principle, there should be many rectangular pictures judged nonrectangular.

\section{METHOD}

\section{Subjects}

The Ss were 16 male and 11 female Harvard University undergraduate and graduate students, who responded to notices placed on bulletin boards around the campus. Subjects were paid \$2 for participating in this and another unrelated experiment. All reported having normal or fully corrected vision.

\section{Stimulus Materials}

The stimulus materials were line drawings of boxes, some of which could have been and some of which could not have been projections of rectangular boxes according to a rule described in Perkins (1968). The rule is that all three angles, $A, B$, and $C$, labeled as in Figs. 1 and 2, must be greater than $90 \mathrm{deg}$, or in a special case that two angles be exactly $90 \mathrm{deg}$. Figure 1 meets this condition and Fig. 2 does not. Another special case, with just one angle of $90 \mathrm{deg}$, is referred to throughout this paper as the "borderline" case. Although, technically, a box picture with one such angle cannot be a central projection of a rectangular box, this case falls exactly on the boundary between the range of rectangular and nonrectangular boxes.

There were 128 cards, 4.5 in. tall $x$ 6.0 in. wide, each displaying one box with its central vertex at the center of the card. The cards were constructed by Xeroxing 16 drawings of boxes of different shapes, drawings done with a .5-mm line, in black ink on white paper. The copies were cut into cards so that each box appeared in eight orientations on eight cards-vertically, rotated $45 \mathrm{deg}$, rotated $90 \mathrm{deg}$, and so on. All 16 boxes shared one angle (Angle $C$ in Figs. 1 and 2) of $120 \mathrm{deg}$, but Angle $A$ varied in each, taking the values $70,80,85,90,95,100,110$, $120,130,140,145,150,155,160$, 165 , and $170 \mathrm{deg}$. The three edges radiating from the central vertex of each box were all of the same length, $3 \mathrm{~cm}$, and the boxes were drawn as orthogonal projections, that is, with no perspective convergence of parallels. Given the fixed Angle $C$ of $120 \mathrm{deg}$, the rule cited in the previous paragraph implies that all the box pictures with Angle A between 90 and $150 \mathrm{deg}$ could be projections of solid rectangular boxes, and the others could not. Accordingly, seven boxes,
70 through $85 \mathrm{deg}$ and 155 through 170 deg, were within the nonrectangular range, the 90- and 150-deg boxes were borderline, and the seven boxes 95 through $145 \mathrm{deg}$ were within the rectangular range. Close to the borderline cases, Angle A was varied by 5 deg rather than $10 \mathrm{deg}$ to permit sharper scrutiny of Ss' behavior in the neighborhood of these critical points.

The cards were arranged in a random order, and then minimally rearranged so that runs of more than three rectangular or borderline cards, or nonrectangular or borderline cards, did not occur. It was decided to present the cards to Ss in this fixed order. The reasons were that handling of the cards would be more convenient, that reversing the order of presentation of the large and homogeneous deck was not very meaningful, and that there were other convenient methods to test for Ss' learning or becoming more oriented to the task as they proceeded through the deck (see results section).

\section{Procedure}

Each S was tested individually. Rectangular and nonrectangular sample pictures-duplicates of the 70and 120-deg normally oriented stimuli-and three-dimensional cardboard models introduced Ss to the idea that boxes could be rectangular or not, and could appear that way in pictures. Ss were seated at a table with their eyes approximately $6 \mathrm{ft}$ from a book stand. In order to orient that stand perpendicular to each S's line of sight, $E$ temporarily attached to the stand a polystyrene brick with the top and longest face painted black. E adjusted the tilt of the stand until $S$ reported that the top face appeared just edge on. $E$ explained to each $S$ the task he was to undertake, and invited $\mathbf{S}$ to ask procedural questions. Ss were urged to attend to the apparent rectangularity of the individual displays and not to expect patterns in the ordering of the stimuli or the like. No one appeared to take this as a cue to do just the opposite. Ss proceeded quickly with their tasks, almost always finishing within $30 \mathrm{~min}$.

Three different testing procedures were utilized for groups of 8,8 , and 11 Ss. Four males and four females followed the "straight" procedure. The stimulus cards were presented one by one, and $S$ indicated verbally whether each box appeared rectangular. Here and in the other two procedures, $\mathrm{E}$ was careful not to look at and judge the cards himself, and $E$ reported that as far as he could determine, repeated running of the experiment did not result in his anticipating Ss' responses. E placed 
each card in one of two piles, depending on Ss' judgments. At the end of the experiment, Ss' decisions were recorded from the piles onto a scoring sheet.

Another eight Ss, four of each sex, participated in the "samples" design. This was conducted exactly as the "straight" design, except that permanently affixed to the left and right sides of the book stand were the 70- and 120-deg samp!es of nonrectangular and rectangular pictures mentioned above. Ss were invited to compare each stimulus card with these samples if they wished; some did so and some did not.

In the final "pairs" design, the deck was reorganized into two stacks of 64 cards, randomly ordered except that the first, second, third, etc., cards in one corresponded to first, second, third, etc., cards in the other of different rectangularity status (rectangular, nonrectangular, or borderline). The cards were presented in pairs, side by side, and Ss were asked to choose which of the pair appeared most rectangular. Eight males and three females participated.

These three procedures, "straight," "samples," and "pairs," were devised with the expectation that they would prompt progressively finer discriminations as to rectangularity. If the "straight" design yielded poor discrimination, then perhaps the

Table 1

Correspondence Between Ss' Responses and Theory

\begin{tabular}{|c|c|c|c|c|c|}
\hline \multirow[b]{2}{*}{$\begin{array}{c}\text { Test } \\
\text { Procedure }\end{array}$} & \multicolumn{2}{|c|}{$\begin{array}{c}\text { Stimuli from } \\
\text { Rectangular } R \text { ange }\end{array}$} & \multicolumn{2}{|c|}{$\begin{array}{c}\text { Stimuli from } \\
\text { Nonrectangular Range }\end{array}$} & \multirow[b]{2}{*}{$\begin{array}{c}\text { Contingency } \\
\text { Coeff } \div .707 \dagger\end{array}$} \\
\hline & $\begin{array}{l}\text { Percent R ect } \\
\text { Judgments }\end{array}$ & SD & $\begin{array}{l}\text { Percent Rect } \\
\text { Judgments }\end{array}$ & SD & \\
\hline $\begin{array}{l}\text { Straight } \\
\text { Samples } \\
\text { Pairs }\end{array}$ & $\begin{array}{l}92 \\
88 \\
94\end{array}$ & $\begin{array}{r}6 \\
16 \\
6\end{array}$ & $\begin{array}{r}19 \\
14 \\
7\end{array}$ & $\begin{array}{r}13 \\
18 \\
9\end{array}$ & $\begin{array}{l}.84^{*} \\
.85 * \\
.88^{*}\end{array}$ \\
\hline
\end{tabular}

†.707 is maximum contingency coefficient for 2 by 2 table.

$* \boldsymbol{p}<.001$

"samples" or "pairs" designs would provide examples of sharp discrimination under favorable circumstances. The "pairs" design was, of course, quite a different sort of task from the other two, calling for a judgment of relative rectangularity. Good performance on "pairs" logically need not imply good performance on the other two.

\section{RESULTS}

Much of the data analysis was based on scores for each $S$ on each of the 16 boxes, scores ranging from 0 to 8 and indicating the number of times out of 8 that an $S$ judged that box in its 8 rotations to be rectangular. For each test procedure, the mean scores of Ss on each box were computed, and these are displayed in Fig. 3. The heavy line in Fig. 3 indicates the theoretical distribution that would occur if all Ss were to discriminate perfectly between

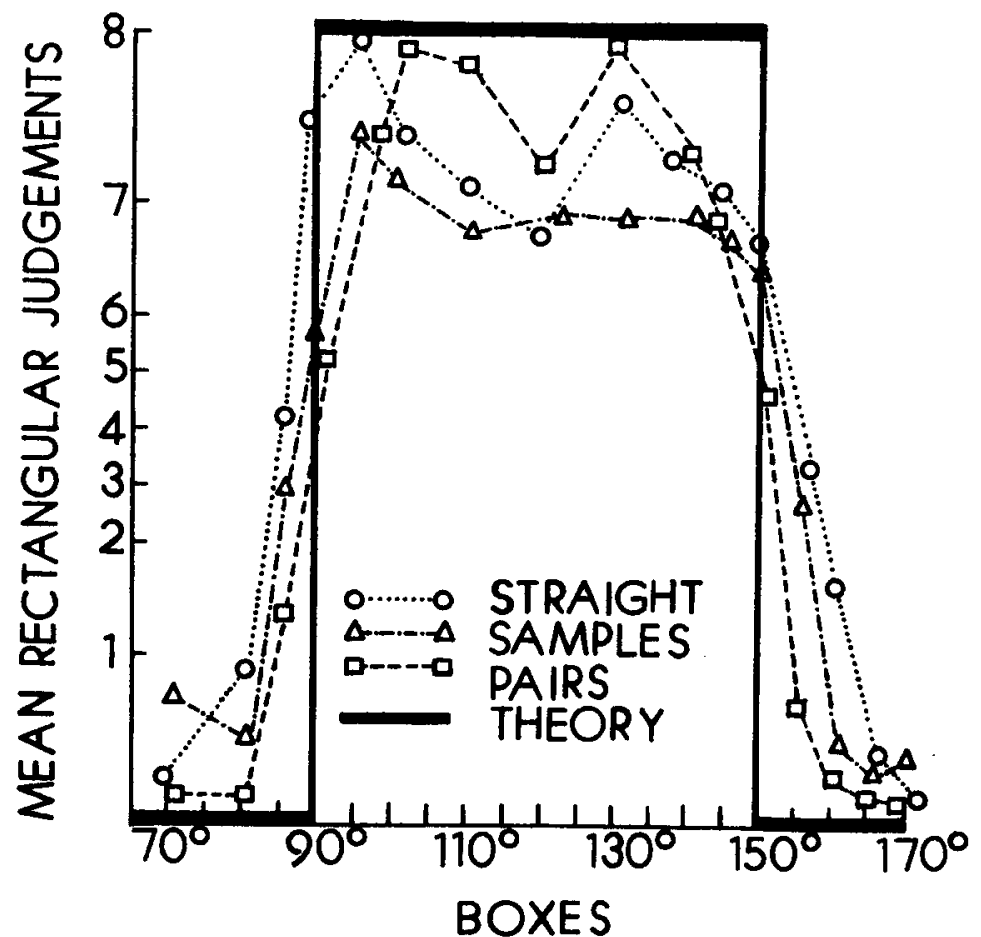

Fig. 3. Mean number of rectangular judgments for each box picture in its eight rotations under each test procedure (the end regions of the ordinate, where data points clustered, are expanded to enhance legibility). rectangular and nonrectangular boxes. The line is discontinuous at 90 and $150 \mathrm{deg}$, reflecting the fact that there is no prediction for the borderline cases. The figure shows clearly that the actual distributions are in close agreement with the hypothesis.

For each test procedure, Table 1 lists percentages of rectangular judgments for the rectangular range and for the nonrectangular range. The null hypothesis that Ss' judgments were not correlated with the theoretical distribution was rejected ( $x^{2}, \mathrm{p}<.001$ for all three conditions). Table 1 lists normalized contingency coefficients calculated from chi square and expressing a correlation between theory and the data.

One question underlying the design of the experiment was whether the discrimination would prove especially sensitive to the circumstances under which it was made. The "samples" or "pairs" conditions might substantially enhance performance. Other experiments using misoriented stimuli (e.g., Kolers \& Perkins, 1969) would lead one to expect at least slight differences in the judgments of the boxes as their orientations varied. Finally, one might anticipate a substantial change in performance as Ss progressed through the deck, becoming more familiar with the task. Computations of skew and kurtosis within test procedures revealed that Ss' scores on the 16 boxes were not at all normally distributed, so nonparametric techniques and two-tailed $t$ tests were employed to detect significant trends. Initial $t$ tests revealed no significant differences between male and female Ss.

As expected, the contingency coefficients of Table 1 show that discrimination under the "pairs" condition was better than it was under "samples," which was better than it was under "straight." But the differences are hardly impressive. To check for significance, the Mann-Whitney $U$ test was applied to the three test procedures in pairs, separately for the rectangular and nonrectangular ranges. For each pair of procedures and range, Ss' scores on each box were normalized and then lumped for the U test. The "pairs" 
procedure was differentiated from "straight" and "samples" in the nonrectangular range $(p<.001$ and .03 , one-tailed).

Data from the "straight" and "samples" conditions were combined to test for orientation and learning effects. For each box in each orientation, the number of the $16 \mathrm{Ss}$ that judged it in accordance with the hypothesis was determined. A Friedmann two-way analysis of variance was performed on these numbers grouped by box, with the eight orientations acting as the different conditions. No significant orientation effect was found $(p>.30)$. To test for improvement as the experiment proceeded, the numbers were rearranged within each group in the order that the rotations of the box occurred in the stimulus deck. Again, the Friedmann statistic proved insignificant $(p>.70)$. In sum, no substantial and systematic differences could be ascribed to variations in test condition, stimulus orientation, or the number of prior stimulus presentations.

However, there were considerable differences in the performance of different Ss, as the high standard deviations of Table 1 suggest. To determine whether Ss were consistent within themselves, Ss' scores on each box were correlated with their scores on every other box, across all 27 Ss. Correlation coefficients between box scores both in the rectangular or both in the nonrectangular ranges were only twice, and insignificantly, negative, and 32 of the 42 coefficients were positive and individually significant $(p<.05)$. Of the 49 coefficients between box scores from the rectangular range and scores from the non rectangular range, none were individually significant and 15 were negative, indicating an overall weak positive trend $(\mathrm{p}<.01$, binomial test, two-tailed). In sum, Ss were consistent within themselves. But the pattern of inter-S differences is best described not as variation along a single "acuity" dimension-which would require significant negative correlations between the rectangular and nonrectangular ranges-but as independent variation in the rectangular and nonrectangular ranges.

The distinct dip at the center of all three of the Fig. 3 curves was a clue to other individual differences. The dip reflected the influence on the means of a minority of individuals within each test procedure, 18 of the $27 \mathrm{Ss}$ scoring at least as well in the center of the rectangular range as toward the boundaries. This minority consistently classified as rectangular only those boxes that were nearly "full face," that is, that presented a frontal facet with a nearly right angle. Their discriminations mixed projective judgments with interpretations appropriate to many children's drawings, as well as to the sophisticated but nonprojective art of several cultures, e.g., early Egyptian (Arnheim, 1967, pp. 93-97).

\section{DISCUSSION}

The data do appear to support the hypothesis described in the introduction as it applies to boxlike figures. As an idealized description, Ss judge box pictures to appear rectangular, (1) only when and (2) always when they could be projections of rectangular boxes. The capacity to make the discrimination varies somewhat from person to person. The findings that judgments were not much affected by varying stimulus orientation, availability of comparisons, and number of prior judgments, suggest that the discrimination is a viable part of a viewer's perceptual repertoire, generally available for his interpreting of his environment.

But it would be misleading to construe a viewer's behavior as directed specifically to rectangular boxes or even corners of such boxes. As remarked at the beginning of this article, typically three organizations of nonrectangular boxes are seen, with alternative pairs of the three angles around the central vertex of the box appearing right. A rectangular organization is accomplished as far as possible short of conflicting with projective possibility. This suggests a much more general mechanism, where right angles as well as, in other examples, different sorts of geometric regularities such as symmetry or support by a ground plane may be read into the stimulus up to the limits allowed by projective possibility (Perkins, 1971).

The impetus toward reading right angles into pictures or real scenes functions in coordination with other gestalt-like organizing principles, as well as with occlusion, texture gradients, and other sorts of depth cues. Normally there will be a considerable concordance in the indications of these various visual means, and this redundancy appears to be at the heart of the visual system's persistent success in making sense of the world. Sometimes a projectively legitimate imposition of rectangularity will be set aside in favor of contrary cues. But in occasional natural or artificial situations, methods will compete to yield an incorrect vote. In Ames's notable experiments with distorted rooms (Ames, 1955, p. 41; Ittelson, 1952, pp. 39-45), naive Ss viewing through a peephole perceived an apparently normal room occupied by people distorted in size. A rectangular interpretation prevailed over knowledge that adult human figures are all about the same size, a sort of knowledge that in other contexts may be put to perceptual use in determining relative distance from ratios of apparent size (Hochberg, 1964 , p. 78 ).

The way the apparently rectangular room altered the appearance of its contents emphasizes that the imposition of rectangular and other geometric organizations is not simply a categorizing game played by the visual system. Applying such constraints logically, and seemingly psychologically, permits subsequent inference of the space configuration represented by a picture or presented by an actual object; and this inference in turn yields further conclusions about other nearby or contained objects. In particular, the equations expressing that a projected corner consists of three right angles can be solved to yield the exact orientation of the corner in space (Perkins, 1968). When an entire box is involved, this information is sufficient then to determine the actual relative proportion of the sides, though not, of course, the absolute size. If perspective convergence effects are significant, even that can be determined.

Although one does not expect the visual system to be solving simultaneous trigonometric equations, apparently it somehow achieves much the same end. A recent study by Attneave and Frost (1969) involved Ss aligning a wand with the apparent orientations of the edges of pictured rectangular boxes. Results show that Ss' alignments were proportional to the theoretical expectation, though with considerable regression toward the plane of the page. The Attneave and Frost study also dealt with pictures of boxes in perspective and not, and with the effect of making the boxes spatially equal sided. Perspective and equal-sidedness substantially improved the accuracy of alignment. The article by Attneave and Frost and the results reported here show the importance of geometric constraints in achieving a spatial interpretation of a visual stimulus.

\section{REFERENCES}

AMES, A.. JR. An interpretative manual for the demonstrations in the Psychology Research Center, Princeton University. The nature of our perceptions, prehensions and behavior. Princeton: Princeton University Press, 1955.

ARNHEIM, R. Art and visual perception: A psychology of the creative eye. Berkeley: University of California Press, 1967.

ATTNEAVE, F., \& FROST, R. The determination of perceived tridimensional orientation by minimum criteria. Perception \& Psychophysics, 1969, 6, 391-396. 
BECK, J., \& GIBSON, J. J. The relation of apparent shape to apparent slant in the perception of objects. Journal of Experimental Psychology, 1955, 50, 125-133.

ERIKSSON, E. S. The shape slant invariance hypothesis in static perception. Scandinavian Journal of Psychology, $1967,8,193-208$

HOCHBERG, J. E. Perception. Englewood Cliffs, N.J: Prentice-Hall, 1964.

I TTELSON, W. H. The Ames demonstrations in perception: A guide to their construction and use. Princeton: Princeton University Press, 1952.

KAISER, $P$. K. Perceived shape and its dependency on perceived slant. Journal of Experimental Psychology, 1967, 75. 345-353.

KOLERS, P. A., \& PERKINS, D. N. Orientation of letters and errors in their recognition. Perception \& Psychophysics. $1969,5,265-269$.

PERKINS, D. N. Cubic comers. In Quarterly Progress Report No.89, Ruarterly Progress Report No. 89,
Lesearch
Massachusetts Institute of Technology, Cambridge, Mass., 1968

PERKINS, D. N. The perception of line drawings of simple space forms. In $D$. N Perkins, Geometry and the perception of pictures: Three studies. Technical Report No. 5, Harvard Project Zero, Graduate School of Education, Harvard University. Cambridge, Mass., 1971.

(Revision received for publication June 19 l972.) 